\title{
INTUITIONISTIC FUZZY ALPHA-CONTINUITY AND INTUITIONISTIC FUZZY PRECONTINUITY
}

\author{
JOUNG KON JEON, YOUNG BAE JUN, AND JIN HAN PARK
}

Received 28 February 2005 and in revised form 20 June 2005

A characterization of intuitionistic fuzzy $\alpha$-open set is given, and conditions for an IFS to be an intuitionistic fuzzy $\alpha$-open set are provided. Characterizations of intuitionistic fuzzy precontinuous (resp., $\alpha$-continuous) mappings are given.

\section{Introduction}

After the introduction of fuzzy sets by Zadeh, there have been a number of generalizations of this fundamental concept. The notion of intuitionistic fuzzy sets introduced by Atanassov is one among them. Using the notion of intuitionistic fuzzy sets, Çoker [5] introduced the notion of intuitionistic fuzzy topological spaces. In this paper, we define the notion of intuitionistic fuzzy semiopen (resp., preopen and $\alpha$-open) mappings and investigate relation among them. We give a characterization of intuitionistic fuzzy $\alpha$-open set, and provide conditions for an IFS to be an intuitionistic fuzzy $\alpha$-open set. We discuss characterizations of intuitionistic fuzzy precontinuous (resp., $\alpha$-continuous) mappings. We give a condition for a mapping of IFTSs to be an intuitionistic fuzzy $\alpha$-continuous mapping.

\section{Preliminaries}

Definition 2.1 (Atanassov [1]). An intuitionistic fuzzy set (IFS) $A$ in $X$ is an object having the form

$$
A=\left\{\left\langle x, \mu_{A}(x), \gamma_{A}(x)\right\rangle \mid x \in X\right\}
$$

where the functions $\mu_{A}: X \rightarrow[0,1]$ and $\gamma_{A}: X \rightarrow[0,1]$ denote the degree of membership (namely, $\mu_{A}(x)$ ) and the degree of nonmembership (namely, $\gamma_{A}(x)$ ) of each element $x \in X$ to the set $A$, respectively, and $0 \leq \mu_{A}(x)+\gamma_{A}(x) \leq 1$ for each $x \in X$.

Definition 2.2 (Atanassov [1]). Let $A$ and $B$ be IFSs of the forms $A=\left\{\left\langle x, \mu_{A}(x), \gamma_{A}(x)\right\rangle \mid\right.$ $x \in X\}$ and $B=\left\{\left\langle x, \mu_{B}(x), \gamma_{B}(x)\right\rangle \mid x \in X\right\}$. Then

(a) $A \subseteq \mathrm{B}$ if and only if $\mu_{A}(x) \leq \mu_{B}(x)$ and $\gamma_{A}(x) \geq \gamma_{B}(x)$ for all $x \in X$,

(b) $A=B$ if and only if $A \subseteq B$ and $B \subseteq A$, 
(c) $\bar{A}=\left\{\left\langle x, \gamma_{A}(x), \mu_{A}(x)\right\rangle \mid x \in X\right\}$,

(d) $A \cap B=\left\{\left\langle x, \mu_{A}(x) \wedge \mu_{B}(x), \gamma_{A}(x) \vee \gamma_{B}(x)\right\rangle \mid x \in X\right\}$,

(e) $A \cup B=\left\{\left\langle x, \mu_{A}(x) \vee \mu_{B}(x), \gamma_{A}(x) \wedge \gamma_{B}(x)\right\rangle \mid x \in X\right\}$.

For the sake of simplicity, we will use the notation $A=\left\langle x, \mu_{A}, \gamma_{A}\right\rangle$ instead of $A=$ $\left\{\left\langle x, \mu_{A}(x), \gamma_{A}(x)\right\rangle \mid x \in X\right\}$. A constant fuzzy set taking value $\alpha \in[0,1]$ will be denoted by $\underline{\alpha}$. The IFSs $0_{\sim}$ and $1_{\sim}$ are defined to be $0_{\sim}=\langle x, \underline{0}, \underline{1}\rangle$ and $1_{\sim}=\langle x, \underline{1}, \underline{0}\rangle$, respectively. Let $\alpha, \beta \in[0,1]$ with $\alpha+\beta \leq 1$. An intuitionistic fuzzy point (IFP), written as $p_{(\alpha, \beta)}$, is defined to be an IFS of $X$ given by

$$
p_{(\alpha, \beta)}(x):= \begin{cases}(\alpha, \beta) & \text { if } x=p \\ (0,1) & \text { otherwise. }\end{cases}
$$

Let $f$ be a mapping from a set $X$ to a set $Y$. If

$$
B=\left\{\left\langle y, \mu_{B}(y), \gamma_{B}(y)\right\rangle: y \in Y\right\}
$$

is an IFS in $Y$, then the preimage of $B$ under $f$, denoted by $f^{-1}(B)$, is the IFS in $X$ defined by

$$
f^{-1}(B)=\left\{\left\langle x, f^{-1}\left(\mu_{B}\right)(x), f^{-1}\left(\gamma_{B}\right)(x)\right\rangle: x \in X\right\}
$$

and the image of $A$ under $f$, denoted by $f(A)$, is an IFS of $Y$ defined by

$$
f(A)=\left\langle y, f\left(\mu_{A}\right), f\left(\gamma_{A}\right)\right\rangle,
$$

where

$$
\begin{aligned}
& f\left(\mu_{A}\right)(y):= \begin{cases}\sup _{x \in f^{-1}(y)} \mu_{A}(x) & \text { if } f^{-1}(y) \neq \varnothing, \\
0 & \text { otherwise, }\end{cases} \\
& f\left(\gamma_{A}\right)(y):= \begin{cases}\inf _{x \in f^{-1}(y)} \gamma_{A}(x) & \text { if } f^{-1}(y) \neq \varnothing, \\
1 & \text { otherwise, }\end{cases}
\end{aligned}
$$

for each $y \in Y$. Çoker [5] generalized the concept of fuzzy topological space, first initiated by Chang [4], to the case of intuitionistic fuzzy sets as follows. 
Definition 2.3 (Çoker [5, Definition 3.1]). An intuitionistic fuzzy topology (IFT) on $X$ is a family $\tau$ of IFSs in $X$ satisfying the following axioms:

(T1) $0_{\sim}, 1_{\sim} \in \tau$,

(T2) $G_{1} \cap G_{2} \in \tau$ for any $G_{1}, G_{2} \in \tau$,

(T3) $\cup G_{i} \in \tau$ for any family $\left\{G_{i} \mid i \in J\right\} \subseteq \tau$.

In this case, the pair $(X, \tau)$ is called an intuitionistic fuzzy topological space (IFTS) and any IFS in $\tau$ is known as an intuitionistic fuzzy open set (IFOS) in $X$. The complement $\bar{A}$ of an IFOS $A$ in IFTS $(X, \tau)$ is called an intuitionistic fuzzy closed set (IFCS) in $X$.

Definition 2.4 (Çoker [5, Definition 3.13]). Let $(X, \tau)$ be an IFTS and let $A=\left\langle x, \mu_{A}, \gamma_{A}\right\rangle$ be an IFS in $X$. Then the intuitionistic fuzzy interior and intuitionistic fuzzy closure of $A$ are defined by

$$
\begin{aligned}
& \operatorname{int}(A)=\bigcup\{G \mid G \text { is an IFOS in } X \text { and } G \subseteq A\}, \\
& \operatorname{cl}(A)=\bigcap\{K \mid K \text { is an IFCS in } X \text { and } A \subseteq K\} .
\end{aligned}
$$

Note that for any IFS $A$ in $(X, \tau)$, we have

$$
\operatorname{cl}(\bar{A})=\overline{\operatorname{int}(A)}, \quad \operatorname{int}(\bar{A})=\overline{\operatorname{cl}(A)} .
$$

\section{Intuitionistic fuzzy openness}

Definition 3.1 [7]. An IFS $A$ in an $\operatorname{IFTS}(X, \tau)$ is called

(i) an intuitionistic fuzzy semiopen set (IFSOS) if

$$
A \subseteq \mathrm{cl}(\operatorname{int}(A)),
$$

(ii) an intuitionistic fuzzy $\alpha$-open set (IF $\alpha \mathrm{OS}$ ) [3] if

$$
A \subseteq \operatorname{int}(\operatorname{cl}(\operatorname{int}(A))),
$$

(iii) an intuitionistic fuzzy preopen set (IFPOS) if

$$
A \subseteq \operatorname{int}(\mathrm{cl}(A)),
$$

(iv) an intuitionistic fuzzy regular open set (IFROS) if

$$
\operatorname{int}(\mathrm{cl}(A))=A \text {. }
$$


An IFS A is called an intuitionistic fuzzy semiclosed set, intuitionistic fuzzy $\alpha$-closed set, intuitionistic fuzzy preclosed set, and intuitionistic fuzzy regular closed set, respectively (IFSCS, IF $\alpha$ CS, IFPCS, and IFRCS, resp.), if the complement of $A$ is an IFSOS, IF $\alpha$ OS, IFPOS, and IFROS, respectively.

In the following diagram, we provide relations between various types of intuitionistic fuzzy openness (intuitionistic fuzzy closedness):

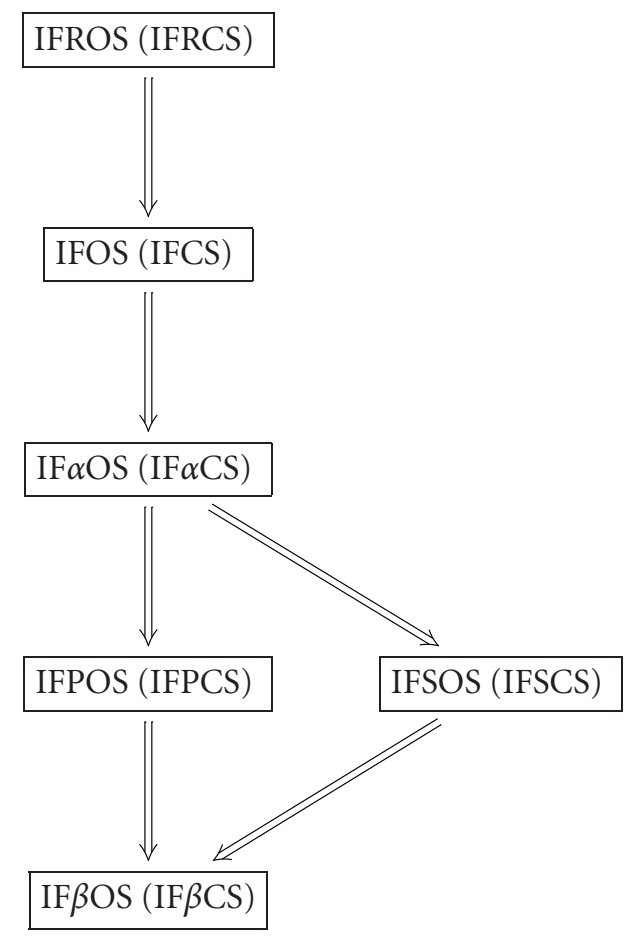

The reverse implications are not true in the above diagram (see [7]). The following is a characterization of an IF $\alpha$ OS.

Theorem 3.2. An IFS A in an IFTS $(X, \tau)$ is an IF $\alpha O S$ if and only if it is both an IFSOS and an IFPOS.

Proof. Necessity follows from the diagram given above. Suppose that $A$ is both an IFSOS and an IFPOS. Then $A \subseteq \operatorname{cl}(\operatorname{int}(A))$, and so

$$
\mathrm{cl}(A) \subseteq \mathrm{cl}(\operatorname{cl}(\operatorname{int}(A)))=\mathrm{cl}(\operatorname{int}(A))
$$

It follows that $A \subseteq \operatorname{int}(\operatorname{cl}(A)) \subseteq \operatorname{int}(\operatorname{cl}(\operatorname{int}(A)))$, so that $A$ is an IF $\alpha \mathrm{OS}$.

We give condition(s) for an IFS to be an IF $\alpha$ OS.

Theorem 3.3. Let $A$ be an IFS in an IFTS $(X, \tau)$. If $B$ is an IFSOS such that $B \subseteq A \subseteq$ $\operatorname{int}(\mathrm{cl}(B))$, then $A$ is an IF $\alpha O S$. 
Proof. Since $B$ is an IFSOS, we have $B \subseteq \mathrm{cl}(\operatorname{int}(B))$. Thus,

$$
A \subset \operatorname{int}(\operatorname{cl}(B)) \subseteq \operatorname{int}(\operatorname{cl}(\operatorname{cl}(\operatorname{int}(B))))=\operatorname{int}(\operatorname{cl}(\operatorname{int}(B))) \subseteq \operatorname{int}(\operatorname{cl}(\operatorname{int}(A)))
$$

and so $A$ is an IF $\alpha$ OS.

LEMma 3.4. Any union of IF $\alpha O S$ s (resp., IFPOSs) is an IF $\alpha O S$ (resp., IFPOS).

The proof is straightforward.

Theorem 3.5. An IFS $A$ in an IFTS $X$ is intuitionistic fuzzy $\alpha$-open (resp., intuitionistic fuzzy preopen) if and only if for every IFP $p_{(\alpha, \beta)} \in A$, there exists an IF $\alpha O S$ (resp., IFPOS) $B_{p_{(\alpha, \beta)}}$ such that $p_{(\alpha, \beta)} \in B_{p_{(\alpha, \beta)}} \subseteq A$.

Proof. If $A$ is an IF $\alpha$ OS (resp., IFPOS), then we may take $B_{p_{(\alpha, \beta)}}=A$ for every $p_{(\alpha, \beta)} \in A$. Conversely assume that for every IFP $p_{(\alpha, \beta)} \in A$, there exists an IF $\alpha \mathrm{OS}$ (resp., IFPOS) $B_{p_{(\alpha, \beta)}}$ such that $p_{(\alpha, \beta)} \in B_{p_{(\alpha, \beta)}} \subseteq A$. Then,

$$
A=\bigcup\left\{p_{(\alpha, \beta)} \mid p_{(\alpha, \beta)} \in A\right\} \subseteq \bigcup\left\{B_{p_{(\alpha, \beta)}} \mid p_{(\alpha, \beta)} \in A\right\} \subseteq A
$$

and so $A=\bigcup\left\{B_{p_{(\alpha, \beta)}} \mid p_{(\alpha, \beta)} \in A\right\}$, which is an IF $\alpha$ OS (resp., IFPOS) by Lemma 3.4.

Definition 3.6. Let $f$ be a mapping from an $\operatorname{IFTS}(X, \tau)$ to an $\operatorname{IFTS}(Y, \kappa)$. Then, $f$ is called

(i) an intuitionistic fuzzy open mapping if $f(A)$ is an IFOS in $Y$ for every IFOS $A$ in $X$,

(ii) an intuitionistic fuzzy $\alpha$-open mapping if $f(A)$ is an IF $\alpha$ OS in $Y$ for every IFOS $A$ in $X$,

(iii) an intuitionistic fuzzy preopen mapping if $f(A)$ is an IFPOS in $Y$ for every IFOS $A$ in $X$,

(iv) an intuitionistic fuzzy semiopen mapping if $f(A)$ is an IFSOS in $Y$ for every IFOS $A$ in $X$.

We have the following implications in which reverse implications are not valid, where "IF" means "intuitionistic fuzzy":

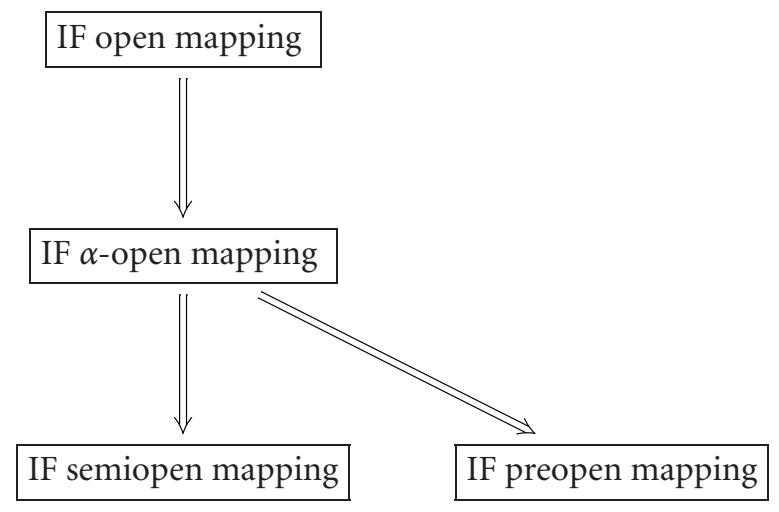


Let $A=\left\langle x, \mu_{A}, \gamma_{A}\right\rangle, B=\left\langle x, \mu_{B}, \gamma_{B}\right\rangle$, and $C=\left\langle x, \mu_{C}, \gamma_{C}\right\rangle$ be IFSs in $I=[0,1]$ defined by

$$
\begin{aligned}
& \mu_{A}(x)=\left\{\begin{array}{ll}
0, & 0 \leq x \leq \frac{1}{2}, \\
2 x-1, & \frac{1}{2} \leq x \leq 1,
\end{array} \quad \gamma_{A}(x)= \begin{cases}1, & 0 \leq x \leq \frac{1}{2}, \\
2(1-x), & \frac{1}{2} \leq x \leq 1,\end{cases} \right. \\
& \mu_{B}(x)=\left\{\begin{array}{ll}
1, & 0 \leq x \leq \frac{1}{4}, \\
2-4 x, & \frac{1}{4} \leq x \leq \frac{1}{2}, \\
0, & \frac{1}{2} \leq x \leq 1,
\end{array} \quad \gamma_{B}(x)= \begin{cases}0, & 0 \leq x \leq \frac{1}{4}, \\
4 x-1, & \frac{1}{4} \leq x \leq \frac{1}{2}, \\
1, & \frac{1}{2} \leq x \leq 1,\end{cases} \right. \\
& \mu_{C}(x)= \begin{cases}0, & 0 \leq x \leq \frac{1}{4}, \\
\frac{1}{3}(4 x-1), & \frac{1}{4} \leq x \leq 1,\end{cases}
\end{aligned}
$$

Then $\tau_{1}=\left\{0_{\sim}, 1_{\sim}, B, A \cup B\right\}, \tau_{2}=\left\{0_{\sim}, 1_{\sim}, \bar{C}\right\}$, and $\tau_{3}=\left\{0_{\sim}, 1_{\sim}, C\right\}$ are IFTSs on $I$. Define a mapping $f: I \rightarrow I$ by $f(x)=\min \{2 x, 1\}$ for each $x \in I$. Then $f\left(0_{\sim}\right)=0_{\sim}, f\left(1_{\sim}\right)=1_{\sim}$, $f(A)=0_{\sim}$, and $f(B)=\bar{A}=f(A \cup B)$. It is easy to verify that $\bar{A}$ is an $\operatorname{IF} \alpha \mathrm{OS}$ in $\left(I, \tau_{2}\right)$. Since $\bar{A} \notin \tau_{2}$, we know that the mapping $f:\left(I, \tau_{1}\right) \rightarrow\left(I, \tau_{2}\right)$ is intuitionistic fuzzy $\alpha$-open which is not intuitionistic fuzzy open. We also note that $\bar{A}$ is an IFSOS but not an IFPOS in $\left(I, \tau_{1}\right)$. Hence, $f:\left(I, \tau_{1}\right) \rightarrow\left(I, \tau_{1}\right)$ is an intuitionistic fuzzy semiopen mapping which is not intuitionistic fuzzy preopen, and so, also not intuitionistic fuzzy $\alpha$-open. Further, $\bar{A}$ is an IFPOS which is not an IFSOS in $\left(I, \tau_{3}\right)$. Therefore, $f:\left(I, \tau_{1}\right) \rightarrow\left(I, \tau_{3}\right)$ is an intuitionistic fuzzy preopen mapping which is not intuitionistic fuzzy semiopen, and thus, also not intuitionistic fuzzy $\alpha$-open.

Theorem 3.7. Let $f:(X, \tau) \rightarrow(Y, \kappa)$ and $g:(Y, \kappa) \rightarrow(Z, \delta)$ be mappings of IFTSs. If $f$ is intuitionistic fuzzy open and $g$ is intuitionistic fuzzy $\alpha$-open (resp., intuitionistic fuzzy preopen), then $g \circ f$ is intuitionistic fuzzy $\alpha$-open (resp., intuitionistic fuzzy preopen).

The proof is straightforward.

Theorem 3.8. A mapping $f:(X, \tau) \rightarrow(Y, \kappa)$ is intuitionistic fuzzy $\alpha$-open if and only if it is intuitionistic fuzzy preopen and intuitionistic fuzzy semiopen.

Proof. Necessity follows from the above second diagram (3.9). Assume that $f$ is intuitionistic fuzzy preopen and intuitionistic fuzzy semiopen and let $A$ be an IFOS in $X$. Then, $f(A)$ is an IFPOS as well as an IFSOS in Y. It follows from Theorem 3.2 that $f(A)$ is an IF $\alpha$ OS so that $f$ is an intuitionistic fuzzy $\alpha$-open mapping. 


\section{Intuitionistic fuzzy continuity}

Definition 4.1 [7]. Let $f$ be a mapping from an $\operatorname{IFTS}(X, \tau)$ to an $\operatorname{IFTS}(Y, \kappa)$. Then $f$ is called an intuitionistic fuzzy precontinuous mapping if $f^{-1}(B)$ is an IFPOS in $X$ for every IFOS $B$ in $Y$.

THEOREM 4.2. For a mapping $f$ from an $\operatorname{IFTS}(X, \tau)$ to an $\operatorname{IFTS}(Y, \kappa)$, the following are equivalent.

(i) $f$ is intuitionistic fuzzy precontinuous.

(ii) $f^{-1}(B)$ is an IFPCS in $X$ for every IFCS B in $Y$.

(iii) $\operatorname{cl}\left(\operatorname{int}\left(f^{-1}(A)\right)\right) \subseteq f^{-1}(\mathrm{cl}(A))$ for every IFS $A$ in $Y$.

Proof. (i) $\Rightarrow$ (ii). The proof is straightforward.

(ii) $\Rightarrow$ (iii). Let $A$ be an IFS in $Y$. Then $\operatorname{cl}(A)$ is intuitionistic fuzzy closed. It follows from (ii) that $f^{-1}(\mathrm{cl}(A))$ is an IFPCS in $X$ so that

$$
\operatorname{cl}\left(\operatorname{int}\left(f^{-1}(A)\right)\right) \subseteq \operatorname{cl}\left(\operatorname{int}\left(f^{-1}(\operatorname{cl}(A))\right)\right) \subseteq f^{-1}(\operatorname{cl}(A)) .
$$

(iii) $\Rightarrow$ (i). Let $A$ be an IFOS in $Y$. Then $\bar{A}$ is an IFCS in $Y$, and so

$$
\operatorname{cl}\left(\operatorname{int}\left(f^{-1}(\bar{A})\right)\right) \subseteq f^{-1}(\operatorname{cl}(\bar{A}))=f^{-1}(\bar{A}) .
$$

This implies that

$$
\begin{aligned}
\overline{\operatorname{int}\left(\operatorname{cl}\left(f^{-1}(A)\right)\right)} & =\operatorname{cl}\left(\overline{\operatorname{cl}\left(f^{-1}(A)\right)}\right)=\operatorname{cl}\left(\operatorname{int}\left(\overline{f^{-1}(A)}\right)\right) \\
& =\operatorname{cl}\left(\operatorname{int}\left(f^{-1}(\bar{A})\right)\right) \subseteq f^{-1}(\bar{A})=\overline{f^{-1}(A)},
\end{aligned}
$$

and thus $f^{-1}(A) \subseteq \operatorname{int}\left(\operatorname{cl}\left(f^{-1}(A)\right)\right)$. Hence $f^{-1}(A)$ is an IFPOS in $X$, and $f$ is intuitionistic fuzzy precontinuous.

Definition 4.3 [9]. Let $p_{(\alpha, \beta)}$ be an IFP of an IFTS $(X, \tau)$. An IFS $A$ of $X$ is called an intuitionistic fuzzy neighborhood (IFN) of $p_{(\alpha, \beta)}$ if there exists an IFOS $B$ in $X$ such that $p_{(\alpha, \beta)} \in$ $B \subseteq A$.

THeOREM 4.4. Let $f$ be a mapping from an $\operatorname{IFTS}(X, \tau)$ to an $\operatorname{IFTS}(Y, \kappa)$. Then the following assertions are equivalent.

(i) $f$ is intuitionistic fuzzy precontinuous.

(ii) For each IFP $p_{(\alpha, \beta)} \in X$ and every IFN $A$ of $f\left(p_{(\alpha, \beta)}\right)$, there exists an IFPOS $B$ in $X$ such that $p_{(\alpha, \beta)} \in B \subseteq f^{-1}(A)$.

(iii) For each IFP $p_{(\alpha, \beta)} \in X$ and every IFN $A$ of $f\left(p_{(\alpha, \beta)}\right)$, there exists an IFPOS $B$ in $X$ such that $p_{(\alpha, \beta)} \in B$ and $f(B) \subseteq A$.

Proof. (i) $\Rightarrow$ (ii). Let $p_{(\alpha, \beta)}$ be an IFP in $X$ and let $A$ be an IFN of $f\left(p_{(\alpha, \beta)}\right)$. Then there exists an IFOS $B$ in $Y$ such that $f\left(p_{(\alpha, \beta)}\right) \in B \subseteq A$. Since $f$ is intuitionistic fuzzy precontinuous, 
we know that $f^{-1}(B)$ is an IFPOS in $X$ and

$$
p_{(\alpha, \beta)} \in f^{-1}\left(f\left(p_{(\alpha, \beta)}\right)\right) \subseteq f^{-1}(B) \subseteq f^{-1}(A) .
$$

Thus (ii) is valid.

(ii) $\Rightarrow$ (iii). Let $p_{(\alpha, \beta)}$ be an IFP in $X$ and let $A$ be an IFN of $f\left(p_{(\alpha, \beta)}\right)$. The condition (ii) implies that there exists an IFPOS $B$ in $X$ such that $p_{(\alpha, \beta)} \in B \subseteq f^{-1}(A)$ so that $p_{(\alpha, \beta)} \in B$ and $f(B) \subseteq f\left(f^{-1}(A)\right) \subseteq A$. Hence (iii) is true.

(iii) $\Rightarrow$ (i). Let $B$ be an IFOS in $Y$ and let $p_{(\alpha, \beta)} \in f^{-1}(B)$. Then $f\left(p_{(\alpha, \beta)}\right) \in B$, and so $B$ is an IFN of $f\left(p_{(\alpha, \beta)}\right)$ since $B$ is an IFOS. It follows from (iii) that there exists an IFPOS $A$ in $X$ such that $p_{(\alpha, \beta)} \in A$ and $f(A) \subseteq B$ so that

$$
p(\alpha, \beta) \in A \subseteq f^{-1}(f(A)) \subseteq f^{-1}(B) .
$$

Applying Theorem 3.5 induces that $f^{-1}(B)$ is an IFPOS in $X$. Therefore, $f$ is intuitionistic fuzzy precontinuous.

Definition 4.5 [7]. Let $f$ be a mapping from an $\operatorname{IFTS}(X, \tau)$ to an $\operatorname{IFTS}(Y, \kappa)$. Then $f$ is called an intuitionistic fuzzy $\alpha$-continuous mapping if $f^{-1}(B)$ is an IF $\alpha \mathrm{OS}$ in $X$ for every IFOS $B$ in $Y$.

THEOREM 4.6. Let $f$ be a mapping from an $\operatorname{IFTS}(X, \tau)$ to an $\operatorname{IFTS}(Y, \kappa)$ that satisfies

$$
\operatorname{cl}\left(\operatorname{int}\left(\operatorname{cl}\left(f^{-1}(B)\right)\right)\right) \subseteq f^{-1}(\operatorname{cl}(B))
$$

for every IFS B in $Y$. Then $f$ is intuitionistic fuzzy $\alpha$-continuous.

Proof. Let $B$ be an IFOS in $Y$. Then $\bar{B}$ is an IFCS in $Y$, which implies from hypothesis that

$$
\mathrm{cl}\left(\operatorname{int}\left(\operatorname{cl}\left(f^{-1}(\bar{B})\right)\right)\right) \subseteq f^{-1}(\operatorname{cl}(\bar{B}))=f^{-1}(\bar{B}) .
$$

It follows that

$$
\begin{aligned}
\overline{\operatorname{int}\left(\operatorname{cl}\left(\operatorname{int}\left(f^{-1}(B)\right)\right)\right)} & =\operatorname{cl}\left(\overline{\operatorname{cl}\left(\operatorname{int}\left(f^{-1}(B)\right)\right)}\right) \\
& =\operatorname{cl}\left(\operatorname{int}\left(\overline{\operatorname{int}\left(f^{-1}(B)\right)}\right)\right) \\
& =\operatorname{cl}\left(\operatorname{int}\left(\operatorname{cl}\left(\overline{f^{-1}(B)}\right)\right)\right) \\
& =\operatorname{cl}\left(\operatorname{int}\left(\operatorname{cl}\left(f^{-1}(\bar{B})\right)\right)\right) \subseteq f^{-1}(\bar{B}) \\
& =\overline{f^{-1}(B)}
\end{aligned}
$$

so that $f^{-1}(B) \subseteq \operatorname{int}\left(\operatorname{cl}\left(\operatorname{int}\left(f^{-1}(B)\right)\right)\right)$. This shows that $f^{-1}(B)$ is an IF $\alpha$ OS in $X$. Hence, $f$ is intuitionistic fuzzy $\alpha$-continuous. 
THEOREM 4.7. Let $f$ be a mapping from an $\operatorname{IFTS}(X, \tau)$ to an $\operatorname{IFTS}(Y, \kappa)$. Then the following assertions are equivalent.

(i) $f$ is intuitionistic fuzzy $\alpha$-continuous.

(ii) For each IFP $p_{(\alpha, \beta)} \in X$ and every IFN A of $f\left(p_{(\alpha, \beta)}\right)$, there exists an IF $\alpha O S B$ such that $p_{(\alpha, \beta)} \in B \subseteq f^{-1}(A)$.

(iii) For each IFP $p_{(\alpha, \beta)} \in X$ and every IFN A of $f\left(p_{(\alpha, \beta)}\right)$, there exists an IF $\alpha O S B$ such that $p_{(\alpha, \beta)} \in B$ and $f(B) \subseteq A$.

Proof. (i) $\Rightarrow$ (ii). Let $p_{(\alpha, \beta)}$ be an IFP in $X$ and let $A$ be an IFN of $f\left(p_{(\alpha, \beta)}\right)$. Then there exists an IFOS $C$ in $Y$ such that $f\left(p_{(\alpha, \beta)}\right) \in C \subseteq A$. Since $f$ is intuitionistic fuzzy $\alpha$-continuous, $B:=f^{-1}(C)$ is an IF $\alpha$ OS and

$$
p_{(\alpha, \beta)} \in f^{-1}\left(f\left(p_{(\alpha, \beta)}\right)\right) \subseteq f^{-1}(C)=B \subseteq f^{-1}(A) .
$$

Thus (ii) is valid.

(ii) $\Rightarrow$ (iii). Let $p_{(\alpha, \beta)}$ be an IFP in $X$ and let $A$ be an IFN of $f\left(p_{(\alpha, \beta)}\right)$. Then there exists an IF $\alpha$ OS $B$ such that $p_{(\alpha, \beta)} \in B \subseteq f^{-1}(A)$ by (ii). Thus, we have $p_{(\alpha, \beta)} \in B$ and $f(B) \subseteq$ $f\left(f^{-1}(A)\right) \subseteq A$. Hence (iii) is valid.

(iii) $\Rightarrow(\mathrm{i})$. Let $B$ be an IFOS in $Y$ and take $p_{(\alpha, \beta)} \in f^{-1}(B)$. Then $f\left(p_{(\alpha, \beta)}\right)$ $\in f\left(f^{-1}(B)\right) \subseteq B$. Since $B$ is an IFOS, it follows that $B$ is an IFN of $f\left(p_{(\alpha, \beta)}\right)$ so from (iii), there exists an IF $\alpha$ OS $A$ such that $p_{(\alpha, \beta)} \in A$ and $f(A) \subseteq B$. This shows that

$$
p_{(\alpha, \beta)} \in A \subseteq f^{-1}(f(A)) \subseteq f^{-1}(B) .
$$

Using Theorem 3.5, we know that $f^{-1}(B)$ is an IF $\alpha \mathrm{OS}$ in $X$, and hence $f$ is intuitionistic fuzzy $\alpha$-continuous.

Combining Theorems 4.6, 4.7, and [8, Theorems 3.12 and 3.13], we have the following characterization of an intuitionistic fuzzy $\alpha$-continuous mapping.

THeOREM 4.8. Let $f$ be a mapping from an $\operatorname{IFTS}(X, \tau)$ to an $\operatorname{IFTS}(Y, \kappa)$. Then the following assertions are equivalent.

(i) $f$ is intuitionistic fuzzy $\alpha$-continuous.

(ii) If $C$ is an IFCS in $Y$, then $f^{-1}(C)$ is an IF $\alpha C S$ in $X$.

(iii) $\operatorname{cl}\left(\operatorname{int}\left(\operatorname{cl}\left(f^{-1}(B)\right)\right)\right) \subseteq f^{-1}(\operatorname{cl}(B))$ for every IFS $B$ in $Y$.

(iv) For each IFP $p_{(\alpha, \beta)} \in X$ and every IFN A of $f\left(p_{(\alpha, \beta)}\right)$, there exists an IF $\alpha O S B$ such that $p_{(\alpha, \beta)} \in B \subseteq f^{-1}(A)$.

(v) For each IFP $p_{(\alpha, \beta)} \in X$ and every IFN $A$ of $f\left(p_{(\alpha, \beta)}\right)$, there exists an IF $\alpha O S B$ such that $p_{(\alpha, \beta)} \in B$ and $f(B) \subseteq A$.

Some aspects of intuitionistic fuzzy continuity, intuitionistic fuzzy almost continuity, intuitionistic fuzzy weak continuity, intuitionistic fuzzy $\alpha$-continuity, intuitionistic fuzzy precontinuity, intuitionistic fuzzy semicontinuity, and intuitionistic fuzzy $\beta$-continuity 
are studied in [7] as well as in several papers. The relation among these types of intuitionistic fuzzy continuity is given in [7] as follows, where "IF" means "intuitionistic fuzzy":

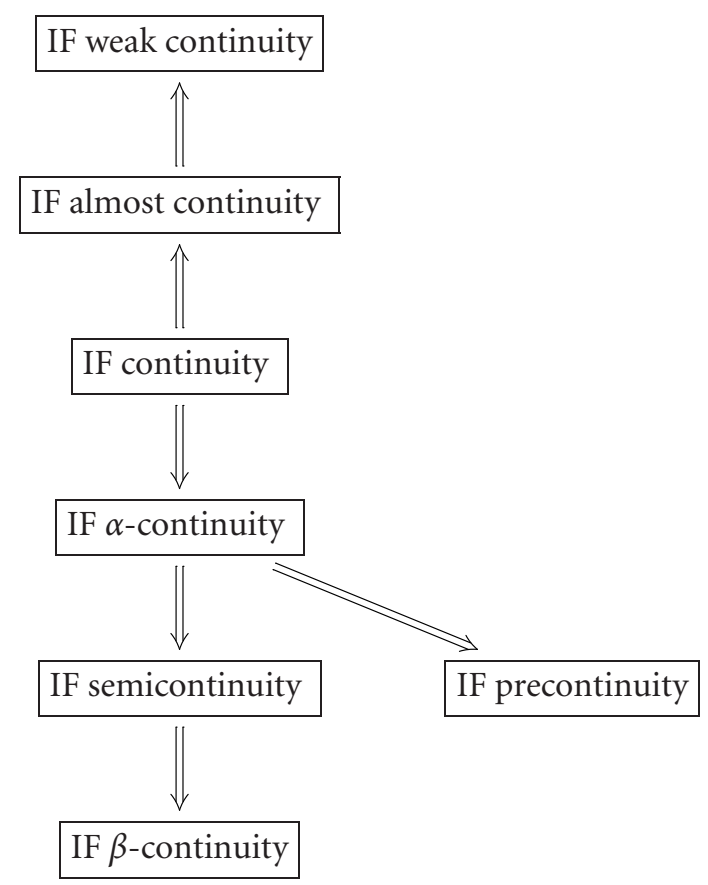

The reverse implications are not true in the above diagram in general (see [7]).

Theorem 4.9. Let $f$ be a mapping from an $\operatorname{IFTS}(X, \tau)$ to an $\operatorname{IFTS}(Y, \kappa)$. If $f$ is both intuitionistic fuzzy precontinuous and intuitionistic fuzzy semicontinuous, then it is intuitionistic fuzzy $\alpha$-continuous.

Proof. Let $B$ be an IFOS in $Y$. Since $f$ is both intuitionistic fuzzy precontinuous and intuitionistic fuzzy semicontinuous, $f^{-1}(B)$ is both an IFPOS and an IFSOS in $X$. It follows from Theorem 3.2 that $f^{-1}(B)$ is an IF $\alpha \mathrm{OS}$ in $X$ so that $f$ is intuitionistic fuzzy $\alpha$-continuous.

\section{Acknowledgments}

The second author, Y. B. Jun, was supported by Korea Research Foundation Grant (KRF2003-005-C00013). The authors are highly grateful to referees for valuable comments and suggestions for improving the paper.

\section{References}

[1] K. T. Atanassov, Intuitionistic fuzzy sets, Fuzzy Sets and Systems 20 (1986), no. 1, 87-96.

[2] K. K. Azad, On fuzzy semicontinuity, fuzzy almost continuity and fuzzy weakly continuity, J. Math. Anal. Appl. 82 (1981), no. 1, 14-32. 
[3] A. S. Bin Shahna, On fuzzy strong semicontinuity and fuzzy precontinuity, Fuzzy Sets and Systems 44 (1991), no. 2, 303-308.

[4] C. L. Chang, Fuzzy topological spaces, J. Math. Anal. Appl. 24 (1968), no. 1, 182-190.

[5] D. Çoker, An introduction to intuitionistic fuzzy topological spaces, Fuzzy Sets and Systems 88 (1997), no. 1, 81-89.

[6] P. Diamond and P. Kloeden, Metric Spaces of Fuzzy Sets. Theory and Applications, World Scientific, New Jersey, 1994.

[7] H. Gürçay, D. Çoker, and A. H. Eş, On fuzzy continuity in intuitionistic fuzzy topological spaces, J. Fuzzy Math. 5 (1997), no. 2, 365-378.

[8] K. Hur and Y. B. Jun, On intuitionistic fuzzy alpha-continuous mappings, Honam Math. J. 25 (2003), no. 1, 131-139.

[9] S. J. Lee and E. P. Lee, The category of intuitionistic fuzzy topological spaces, Bull. Korean Math. Soc. 37 (2000), no. 1, 63-76.

[10] N. Palaniappan, Fuzzy Topology, CRC Press, Florida; Narosa, New Delhi, 2002.

[11] S. S. Thakur and S. Singh, On fuzzy semi-preopen sets and fuzzy semi-precontinuity, Fuzzy Sets and Systems 98 (1998), no. 3, 383-391.

Joung Kon Jeon: Department of Mathematics Education, College of Education, Gyeongsang National University, Jinju 660-701, Korea

E-mail address: jjk5776@hanmail.net

Young Bae Jun: Department of Mathematics Education, College of Education, Gyeongsang National University, Jinju 660-701, Korea

E-mail address: ybjun@gsnu.ac.kr

Jin Han Park: Division of Mathematical Sciences, College of Natural Sciences, Pukyong National University, Pusan 608-737, Korea

E-mail address: jihpark@pknu.ac.kr 


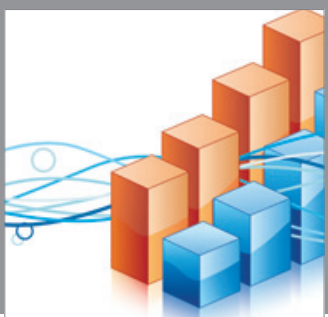

Advances in

Operations Research

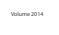

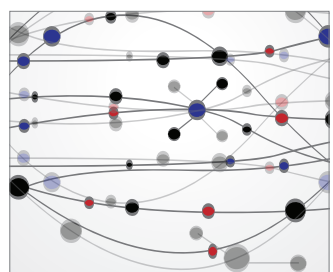

\section{The Scientific} World Journal
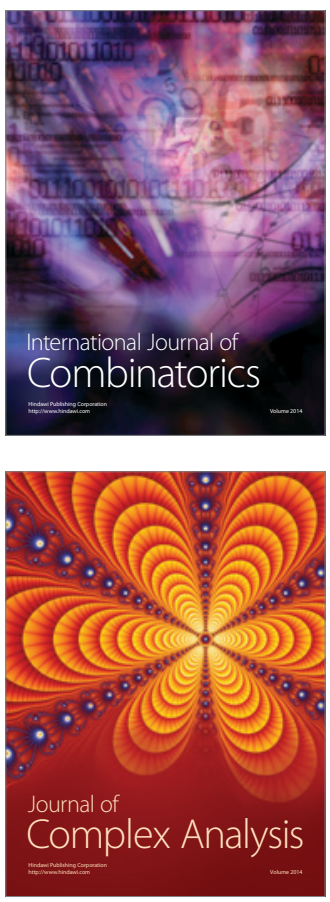

International Journal of

Mathematics and

Mathematical

Sciences
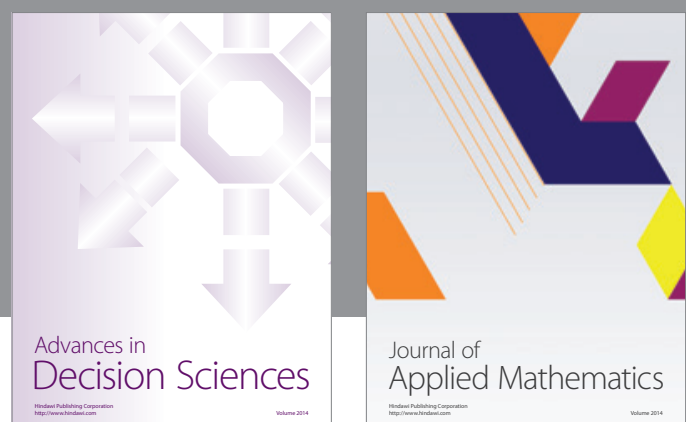

Journal of

Applied Mathematics
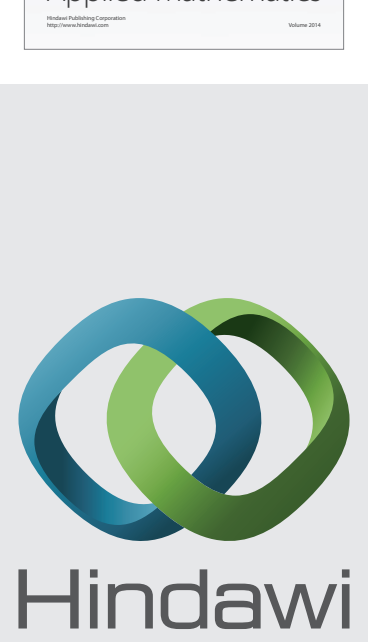

Submit your manuscripts at http://www.hindawi.com
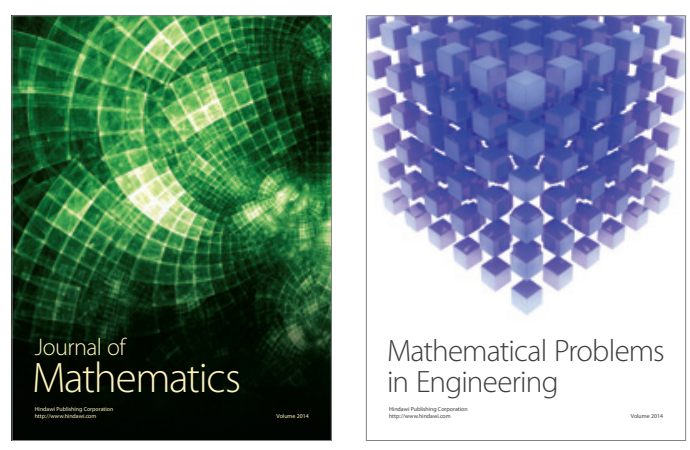

Mathematical Problems in Engineering
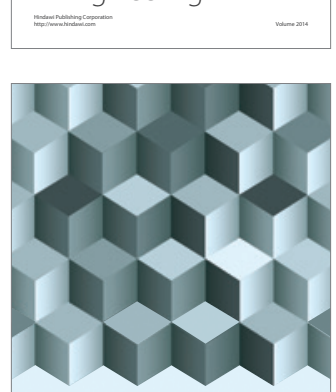

Journal of

Function Spaces
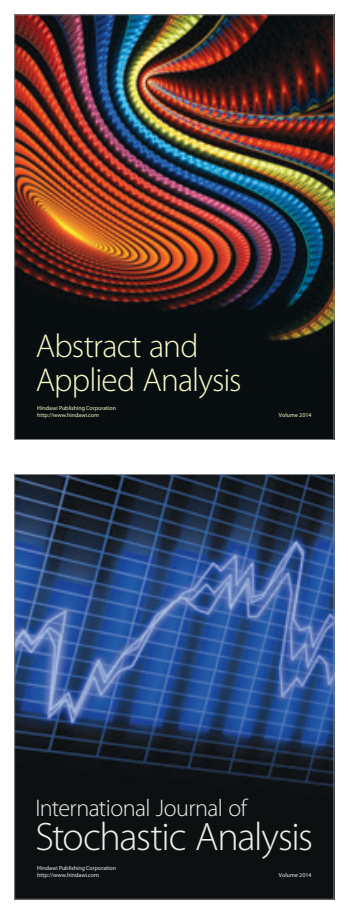

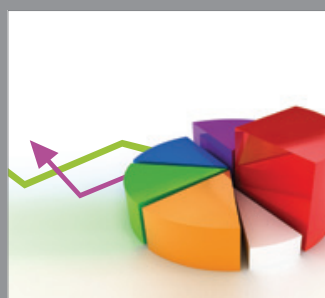

ournal of

Probability and Statistics

Promensencen
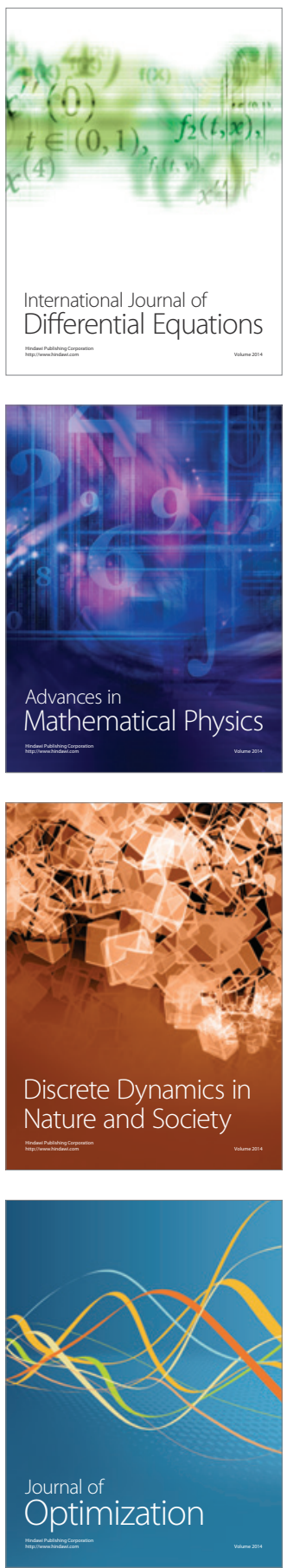